\title{
NON-UNIFORM SAMPLING AND GAUSSIAN PROCESS REGRESSION IN TRANSPORT OF INTENSITY PHASE IMAGING
}

\author{
Zhong Jingshan ${ }^{1,2}$, Rene A. Claus ${ }^{3}$, Justin Dauwels ${ }^{1}$, Lei Tian ${ }^{2}$, Laura Waller $^{2}$ \\ ${ }^{1}$ School of Electrical and Electronic Engineering, Nanyang Technological University, Singapore 639798 \\ ${ }^{2}$ Department of Electrical Engineering and Computer Sciences, University of California, Berkeley \\ ${ }^{3}$ Applied Science and Technology, University of California, Berkeley
}

\begin{abstract}
Gaussian process (GP) regression is a nonparametric regression method that can be used to predict continuous quantities. Here, we show that the same technique can be applied to a class of phase imaging techniques based on measurements of intensity at multiple propagation distances, i.e. the transport of intensity equation (TIE). In this paper, we demonstrate how to apply GP regression to estimate the first intensity derivative along the direction of propagation and incorporate nonuniform propagation distance sampling. The low-frequency artifacts that often occur in phase recovery using traditional methods can be significantly suppressed by the proposed GP TIE method. The method is shown to be stable with moderate amounts of Gaussian noise. We validate the method experimentally by recovering the phase of human cheek cells in a bright field microscope and show better performance as compared to other TIE reconstruction methods.
\end{abstract}

Index Terms - Phase imaging, Gaussian process

\section{INTRODUCTION}

Quantitative phase imaging has useful applications in biology and surface metrology [1, 2], since objects of interest often do not absorb light, but cause phase delays. Phase cannot be directly measured by a camera. It needs to be reconstructed from intensity images. Phase retrieval methods using a series of intensity images measured through focus [3-7] are especially interesting, because they have the advantage of simple experimental setup (Fig. 1). The setup in Fig. 11is widely used in microscopes. A stack of defocused intensity images can be obtained by moving the camera.

The transport of intensity equation (TIE) [3-5] is a class of popular methods to recover phase from defocused intensity images. Besides of simplicity in experimental setup, the TIE method is advantageous because it is valid for partially coherent illumination [8, 9]. Interestingly, when higher-order coherence functions need to be recovered, phase space tomography provides an analogous procedure [10]. The transport of

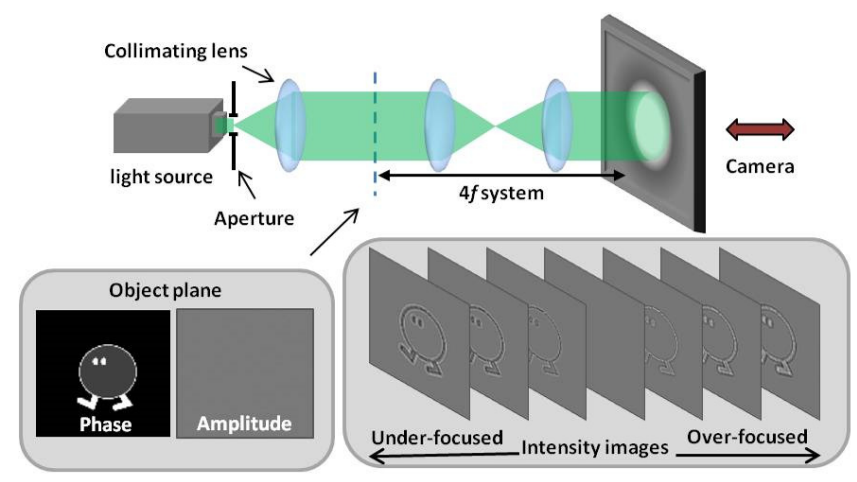

Fig. 1. Experimental setup. A microscope captures intensity images at various defocus distances in order to recover phase.

intensity equation is [3]:

$$
\left.\frac{\partial I(x, y, z)}{\partial z}\right|_{z=0}=-\frac{\lambda}{2 \pi} \nabla_{\perp} \bullet\left(I(x, y) \nabla_{\perp} \phi(x, y)\right),
$$

where $I(x, y)$ is the intensity at the focus, $\phi(x, y)$ is the phase, $\lambda$ is the spectrally-weighted mean wavelength of illumination, and $\nabla_{\perp}$ denotes the gradient operator in lateral directions $(x, y)$ only. It reveals the relationship between phase and the first derivative of intensity with respect to the optical axis, $z$, at focus $(z=0)$. When $I(x, y)$ is constant (i.e. a pure phase object), the phase is solved with Laplacian inversion

$$
\Phi(u, v)=F(u, v) /\left(-4 \pi^{2}\left(u^{2}+v^{2}\right)\right),
$$

where $\Phi(u, v)$ is the Fourier transform of $\phi(x, y), F(u, v)$ is the Fourier transform of the first derivative $\left.\frac{\partial I(x, y, z)}{\partial z}\right|_{z=0}$ scaled by $\frac{2 \pi}{\lambda}$, and $u, v$ are the spatial frequency variables. When $\mathrm{I}(\mathrm{x}, \mathrm{y})$ is not constant, a similar approach involving two Laplacian inversions must be used.

The main limitations of the TIE method are nonlinearity in the intensity derivative estimate and noise, both of which we address here. Intensity derivatives cannot be measured directly and so must be estimated from finite difference methods, which subtract two images near focus. Since intensity is not linear through focus, due to diffraction, this approach 
unavoidably has nonlinearity error error. This error can be reduced by using a higher order TIE [5], which performs polynomial fitting on multiple intensity images. However, the noise in the images means that there is a danger of overfitting. The Savitzky-Golay differentiation filter (SGDF) TIE [11] was proposed to solve this trade-off between the order of fitting and noise in higher order TIE. It recovers phase images with different orders of polynomial fitting, then combines these phase images into a final reconstructed phase. However, the fitting becomes unstable when the order is large [12].

The popular scheme of measuring equally spaced intensity images along the propagation direction, $z$, in higher TIE and SGDF TIE is not ideal. An optimal step size for two consecutive images $(d z)$ is difficult to find. First, it depends on the spatial spectrum of the object and the noise statistics of the camera [13]. Second, there is a trade-off between large and small step size, $d z$. Measuring the low-frequency information of phase is particularly important, because the Laplacian inversion in Eq. (2) amplifies low-frequency error. A large $d z$ is required to transfer the low-frequency information of phase into the intensity images. However, the high-frequency components favour a small $d z$. Instead of trading off, we adopt here a nonequal spacing scheme for multiple defocus planes, which takes both low and high frequency into account.

We propose a TIE phase recovery method using Gaussian process regression [14] to deal with issues of nonlinearity, noise and nonequal spacing. Our method performs regression for each frequency component of the Fourier transform of intensity images. Instead of doing a pure numerical approximation, the proposed GP TIE method incorporates the property of free-space wave propagation in the regression.

This paper is structured as follows. In Section 2, we introduce the basic theory of Gaussian process regression. In Section 3, we apply the Gaussian process regression to TIE phase recovery. In Section 4 we propose a nonequal spacing measurement scheme. In Section 5, we show the experimental result. We offer concluding remarks in Section 6 .

\section{GAUSSIAN PROCESS REGRESSION}

We review the basics of Gaussian process regression. Consider the problem of 2-D regression, given input/output pairs $\left(z_{n}, f_{n}\right)$, where $n=1, \ldots, N$. Under the Gaussian process assumption [14], the outputs $f_{n}$ are drawn from the zero-mean Gaussian distribution with the covariance as a function of $z_{n}$ :

$$
\left(f_{1}, f_{2}, \ldots, f_{N} \mid z_{1}, z_{2}, \ldots, z_{N}\right) \sim \mathcal{N}\left(\mathbf{0}, \mathbf{K}(\mathbf{Z}, \mathbf{Z})+\sigma_{n} \mathbf{I}\right)
$$

where $\mathbf{K}(\mathbf{Z}, \mathbf{Z})$ is the covariance matrix of the outputs given the input set $\mathbf{Z}$, and $\sigma_{n}$ is the variance of additive Gaussian noise in outputs. Generally, the squared exponential covariance function is used to model the covariance matrix:

$$
\mathbf{K}_{i j}=\sigma_{f}^{2} \exp \left[-\frac{1}{2 \ell^{2}}\left(z_{i}-z_{j}\right)^{2}\right]
$$

where $\sigma_{f}, \ell$, and $\sigma_{n}$ in Eq. (3) are the hyper-parameters of the GP model. We can write the joint distribution of the observed outputs with the unknown value of $f_{*}$ at point $z_{*}$ as:

$$
\left[\begin{array}{c}
\mathbf{f} \\
f_{*}
\end{array}\right] \sim \mathcal{N}\left(\mathbf{0},\left[\begin{array}{cc}
\mathbf{K}(\mathbf{Z}, \mathbf{Z})+\sigma_{n} \mathbf{I} & \mathbf{K}\left(\mathbf{Z}, z_{*}\right) \\
\mathbf{K}\left(z_{*}, \mathbf{Z}\right) & \mathbf{K}\left(z_{*}, z_{*}\right)
\end{array}\right]\right),
$$

where $\mathbf{f}$ denotes the set of observed outputs. The conditional distribution of the unknown output $f_{*}$ at $z_{*}$ is calculated as

$$
\left(f_{*} \mid f_{1}, f_{2}, \ldots, f_{N}, z_{1}, z_{2}, \ldots, z_{N}, z_{*}\right) \sim \mathcal{N}(\mu, \overline{\mathbf{K}})
$$

where

$$
\begin{aligned}
\mu & =\mathbf{K}\left(z_{*}, \mathbf{Z}\right)^{T}\left(\mathbf{K}(\mathbf{Z}, \mathbf{Z})+\sigma_{n} \mathbf{I}\right)^{-1} \mathbf{f} \\
\overline{\mathbf{K}} & =\mathbf{K}\left(z_{*}, z_{*}\right)-\mathbf{K}\left(z_{*}, \mathbf{Z}\right)\left(\mathbf{K}(\mathbf{Z}, \mathbf{Z})+\sigma_{n} \mathbf{I}\right)^{-1} \mathbf{K}\left(\mathbf{Z}, z_{*}\right)
\end{aligned}
$$

We can define a vector $h\left(z_{*}\right)$ :

$$
h\left(z_{*}\right)=\mathbf{K}\left(z_{*}, \mathbf{Z}\right)^{T}\left(\mathbf{K}(\mathbf{Z}, \mathbf{Z})+\sigma_{n} \mathbf{I}\right)^{-1} .
$$

Thus we have $\mu=h\left(z_{*}\right) \mathbf{f}$. The prediction $\mu$ is a linear combination of the target values $\mathbf{f}$. GP regression can be viewed as a linear smoother, and $h\left(z_{*}\right)$ is called weight function or the equivalent kernel [14]. In [15], the Fourier transform of the weight function for the squared exponential kernel is given as

$$
\tilde{h}_{S E}(\mathbf{s})=\frac{1}{1+b \exp \left(2 \pi^{2} \ell^{2}|\mathbf{s}|^{2}\right)},
$$

where $b=\sigma_{n}^{2} / \rho\left(2 \pi \ell^{2}\right)^{1 / 2}$ and $\rho$ is the average number of observations per unit (for example length). When $b$ is small, $\tilde{h}_{S E}(\mathbf{s})$ is approximated by a step function. The rapid change from 1 to 0 happens at the point when

$$
s_{c}^{2}=\log (1 / b) /\left(2 \pi^{2} \ell^{2}\right) .
$$

Therefore, when $b$ is small, GP regression can be viewed as a low pass filter with $3 d B$ cut-off frequency at $s_{c}[14,15]$.

\section{TIE PHASE RECOVERY USING GAUSSIAN PROCESS REGRESSION}

TIE methods need to estimate $\left.\frac{\partial I(x, y, z)}{\partial z}\right|_{z=0}$ from a stack of intensity images $I\left(x, y, z_{1}\right), I\left(x, y, z_{2}\right), \ldots, I\left(x, y, z_{N}\right)$. Suppose the complex field at $z=0$ is written as

$$
A(x, y, 0)=\exp [i \phi(x, y)-\mu(x, y)],
$$

where $\mu(x, y)=\ln I(x, y)$ describes absorption and $\phi(x, y)$ is the phase. From the weak phase object approximation [16[18], in frequency domain we have

$$
\begin{aligned}
\mathcal{I}(u, v, z)= & \delta-2 U(u, v) \cos \left[\pi \lambda\left(u^{2}+v^{2}\right) z\right], \\
& -2 \Phi(u, v) \sin \left[\pi \lambda\left(u^{2}+v^{2}\right) z\right]
\end{aligned}
$$


where $\delta$ denotes Dirac delta function, and $U(u, v), \Phi(u, v)$, and $\mathcal{I}(u, v, z)$ are Fourier transform of $\mu(x, y), \phi(x, y)$ and $I(x, y, z)$, respectively.

We perform Gaussian process regression on data points $\mathcal{I}\left(u_{m}, v_{n}, z_{1}\right), \mathcal{I}\left(u_{m}, v_{n}, z_{2}\right), \ldots, \mathcal{I}\left(u_{m}, v_{n}, z_{N}\right)$, for the frequency component $\left(u_{m}, v_{n}\right)$. GP regression does not require the measuring positions $z_{1}, z_{2}, \ldots, z_{N}$ to be equally spaced. We use the low-pass filter property of GP regression by setting appropriate hyper-parameters $\sigma_{f}, \sigma_{n}$, and $\ell$. The hyperparameters $\sigma_{f}$ and $\sigma_{n}$ are initialized to keep $b$ in Eq. (10) small. Then we set the cut-off frequency of the GP regression $s_{c}$ as $\pi \lambda\left(u_{m}^{2}+v_{n}^{2}\right)$ by solving $\ell$ from Eq. (11). The cut-off frequency $s_{c}$ can be larger than $\pi \lambda\left(u_{m}^{2}+v_{n}^{2}\right)$ to allow trade-off of accuracy and noise filtering. From Eqs. (7) 9) we have

$$
\begin{aligned}
\mathcal{I}\left(u_{m}, v_{n}, z\right) & \approx h\left(u_{m}, v_{n}, z\right) \mathbf{I}_{m n}, \\
\left.\frac{\partial \mathcal{I}\left(u_{m}, v_{n}, z\right)}{\partial z}\right|_{z=0} & \left.\approx \frac{\partial h\left(u_{m}, v_{n}, z\right)}{\partial z}\right|_{z=0} \mathbf{I}_{m n},
\end{aligned}
$$

where $\mathbf{I}_{m n}=\left[\mathcal{I}\left(u_{m}, v_{n}, z_{1}\right), \ldots, \mathcal{I}\left(u_{m}, v_{n}, z_{N}\right)\right]^{T}$. After the first derivative of intensity is obtained with Eq. (15), the phase can be recovered with the Laplacian inversion in Eq. (1) (2).

\section{NONEQUAL SPACING MEASUREMENT SCHEME}

We propose a nonequal spacing measurement scheme of the defocused intensity images in order to transfer more phase information to intensity variation for higher SNR. It guarantees that all frequency components of phase are transferred at least by a ratio $\alpha$ to one of the intensity images. From Eq. (13), the phase transfer function at defocus distance $z$ is expressed as

$$
g(z)=\sin \left[\pi \lambda\left(u^{2}+v^{2}\right) z\right] .
$$

The phase transfer function describes the ratio of phase transferred into intensity for each frequency component. Suppose we start from a minimum measuring distance $z_{1}$, and the aim is to find the next $z_{2}$ larger than $z_{1}$. Figure 2 shows the transfer functions $g\left(z_{1}\right)$ and $g\left(z_{2}\right)$ using $\pi \lambda\left(u^{2}+v^{2}\right)$ as x-axis. If the first intersection point is at $g\left(z_{1}\right)=g\left(z_{2}\right)=\alpha$, then we have $z_{2}=\beta z_{1}$, where $\beta=(\pi-\arcsin (\alpha)) / \arcsin (\alpha)$. The rate $\beta$ increases when the transferring ratio $\alpha$ drops. Following the same rule, we would find a series of measuring points having $z_{n+1}=\beta z_{n}$. Since $z_{n}$ grows exponentially according to $z_{n+1}=\beta z_{n}$, a large $z$ can be efficiently reached, which is crucial for transferring low-frequency phase information.

\section{RESULTS}

We consider three sets of data to assess the performance of Gaussian process TIE phase recovery. Data Set 1 has 9 intensity images artificially generated by moving the camera with a constant step size of $5 \mu \mathrm{m}$ and illumination wavelength of $632.8 \mathrm{~nm}$. Data set 2 consists of 9 simulated intensity images which are captured symmetrically over the focus at

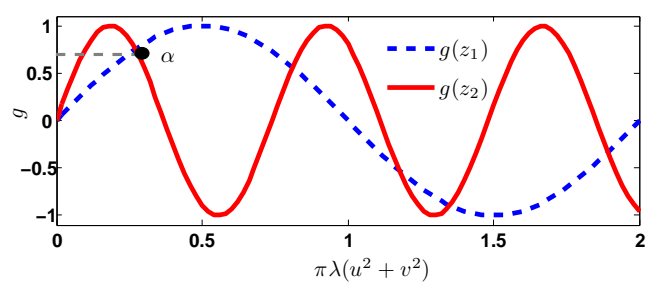

Fig. 2. The phase transfer functions $g\left(z_{1}\right)$ and $g\left(z_{2}\right)$ have the first intersection point at $\alpha$.

$5 \mu m, 20 \mu m, 80 \mu m$, and $320 \mu m$. Each image in Data Set 1 and Data Set 2 has $100 \times 100$ pixels (size $2 \mu m \times 2 \mu m$ ), corrupted by white Gaussian noise of variance 0.006 . Data Set 3 comprises 9 images $(350 \times 360$ pixels with size of $0.62 \mu \mathrm{m} \times 0.62 \mu \mathrm{m})$ of human cheek cells captured with a microscope. The illumination is obtained by filtering white light with a color filter (center wavelength $650 \mathrm{~nm}, 10 \mathrm{~nm}$ bandwidth). The images are captured symmetrically thorough the focus at $5.7 \mu \mathrm{m}, 11.4 \mu \mathrm{m}, 22.8 \mu \mathrm{m}$, and $45.6 \mu \mathrm{m}$. Figure 3 shows the images of simulated data (Data Set 1 and Data Set 2) and experimental data (Data Set 3).
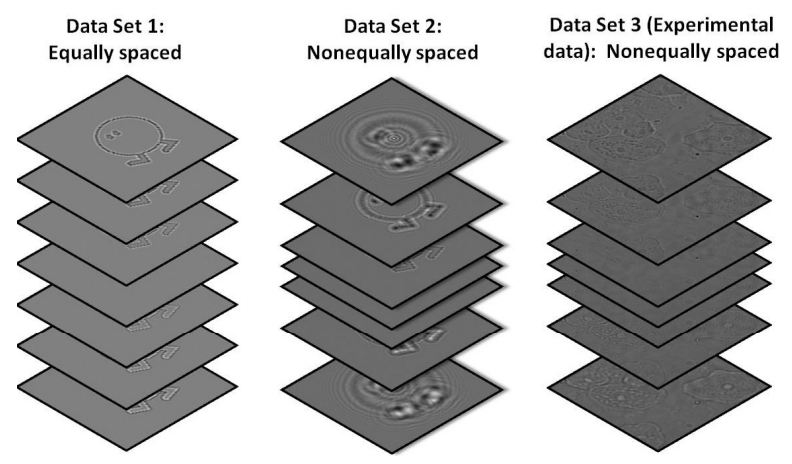

Fig. 3. The measured intensity images of simulated data (Data Set 1 and Data Set 2) and experimental data (Data Set 3).

\subsection{Simulated Data}

We compare the result of higher order TIE [5], SGDF TIE [11], and our proposed GP TIE. Figure 4(a) shows the recovered phase using Data Set 1 , which uses equally spaced planes. For a fair comparison, we show the result of the higher order TIE with the order of polynomial fitting $m=5$, which has best error performance among different orders of fitting. The phase of higher order TIE still has stronger lowfrequency noise compared to SGDF TIE and GP TIE. Figure 4(b) shows the phase recovered by GP and higher order TIE using Data Set 2, which is nonequally spaced. SGDF TIE does not fit to the framework of nonequal spacing, and so is not shown. Higher order TIE can be extended [19] to the case of nonequal spacing. GP TIE recovers a high contrast phase with an average mean square error (MSE) of 0.0065 . We can hardly see any milky low-frequency noise in the 
phase result of GP TIE. The phase recovered by higher order TIE in Fig. 4(b) looks blurred, because it sacrifices the high-frequency information to obtain robust low-frequency recovery. However, GP TIE still returns a sharp phase image.

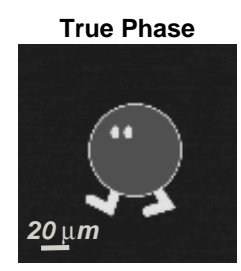

SGDF

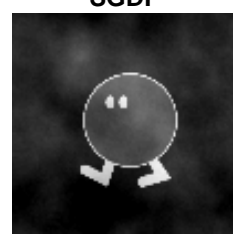

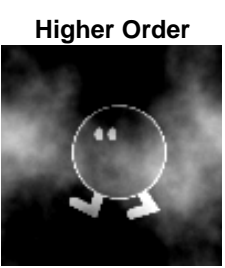

GP

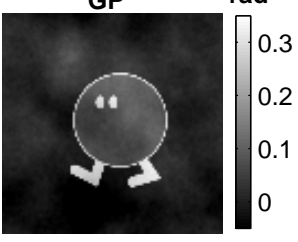

(a)

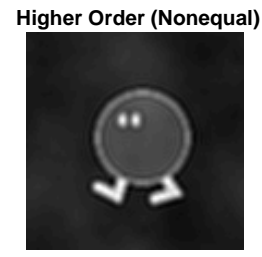

GP (Nonequal)

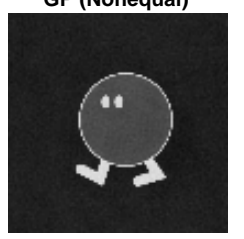

(b)
Fig. 4. (a) Recovered phase images of equally spaced Data Set 1, using higher order TIE (MSE 0.1194 in radian), SGDF TIE (0.0295), and the proposed GP TIE (0.0279). (b) Recovered phase images of nonequally spaced Data Set 2, using higher order TIE (MSE 0.0237) and GP TIE (0.0065).

Figure 5 compares the MSE of recovered phase using different levels of noise. The true intensity images of Data Set 1 and Data Set 2 are corrupted by white Gaussian noise of variance ranging for 0 to 0.2 . We average the MSE over 50 trials. We compare higher order TIE, SGDF TIE and GP TIE with equal and nonequal spacing. Fig.5 shows that GP TIE has slightly lower MSE than SGDF TIE for Data Set 1. For Data Set 2, the MSE is relatively low for higher order TIE of $m=5$ while it is substantially larger for $m=9$, highlighting the importance of choosing the appropriate order, which is difficult since it is object-dependent; on the other hand, GP TIE clearly exhibits the lowest MSE. This can be explained by the fact that Data Set 2 contains more information about low frequency phase than Data Set 1 . The Laplacian inversion is sensitive to low frequency noise in the estimation of the intensity derivative. GP regression using Data Set 2 yields a more robust approximation of the intensity derivative, especially for low-frequency components in the images.

\subsection{Experimental data}

Figure 6 shows the recovered phase of human cheek cells using higher order TIE and GP TIE. We show the results for higher order TIE with the order of polynomial fitting $m$ equal to 2,3 , and 4 . The phase images of $m=2$ and 3 have small low-frequency noise but look blurred. The phase of $m=4$ has high contrast in some regions, but is polluted by lowfrequency noise. However, the phase recovered by GP TIE

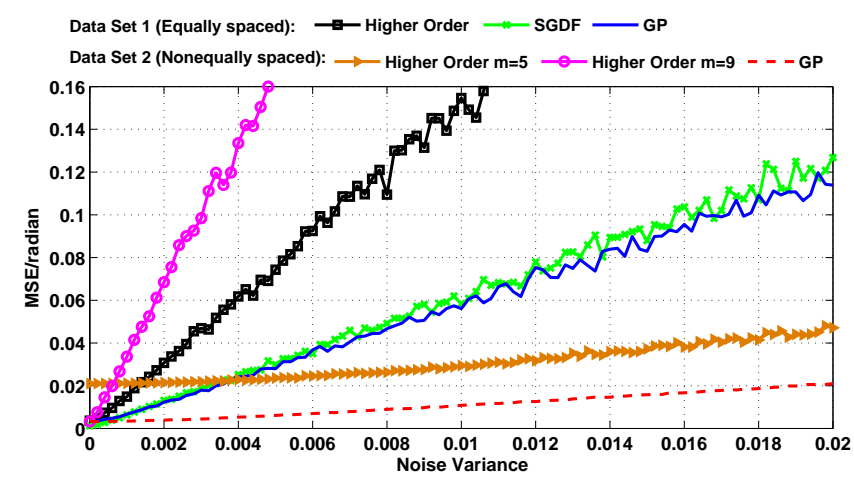

Fig. 5. Comparison of MSE for phase recovery with image stacks polluted by different amounts of white Gaussian noise. GP TIE of nonequally spaced data shows a stable error plot.
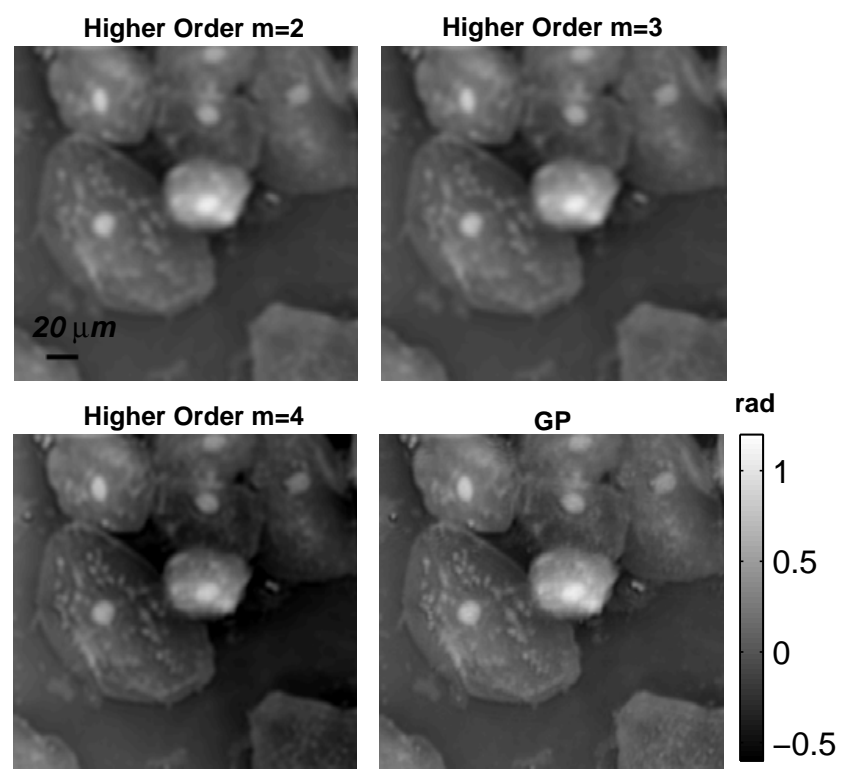

Fig. 6. Phase images of human cheek cells recovered by higher order TIE ( $m=2,3$, and 4 ) and GP TIE.

has less low-frequency noise and high contrast. We can see detail of cells in the phase image recovered by GP TIE.

\section{CONCLUSIONS}

In this paper, we propose a TIE phase recovery method using Gaussian process regression. By incorporating the prior knowledge of wave propagation into the regression, our proposed method recovers phase from intensity images measured in unequally spaced defocus planes. It is robust and stable with different levels of white Gaussian noise. With the freedom of measurement positions, TIE methods can extend to developing new measurement strategies if any prior knowledge of the phase spectrum is known. 


\section{References}

[1] G. Popescu, Quantitative phase imaging of cells and tissues, McGraw-Hill New York, 2011.

[2] K. A. Nugent, D. Paganin, and T. E. Gureyev, "A phase odyssey," Physics Today, vol. 54, no. 8, pp. 27-32, 2001.

[3] M. R. Teague, "Deterministic phase retrieval: a greens function solution," Journal of the Optical Society of America, vol. 73, no. 11, pp. 1434-1441, 1983.

[4] N. Streibl, "Phase imaging by the transport equation of intensity," .

[5] L. Waller, L. Tian, and G. Barbastathis, "Transport of intensity phase-amplitude imaging with higher order intensity derivatives," Optics Express, vol. 18, no. 12, pp. 12552-12561, 2010.

[6] L. Waller, M. Tsang, S. Ponda, S.Y. Yang, and G. Barbastathis, "Phase and amplitude imaging from noisy images by Kalman filtering," Optics Express, vol. 19, no. 3, pp. 2805-2814, 2011.

[7] Z. Jingshan, J. Dauwels, M. A. Vázquez, and L. Waller, "Sparse ACEKF for phase reconstruction," Optics Express, vol. 21, no. 15, pp. 18125-18137, 2013.

[8] D. Paganin and K. A. Nugent, "Noninterferometric phase imaging with partially coherent light," Physical review letters, vol. 80, no. 12, pp. 2586, 1998.

[9] J. C. Petruccelli, L. Tian, and G. Barbastathis, "The transport of intensity equation for optical path length recovery using partially coherent illumination," Optics Express, vol. 21, no. 12, pp. 14430-14441, 2013.

[10] L. Tian, J. Lee, S. B. Oh, and G. Barbastathis, "Experimental compressive phase space tomography," Optics Express, vol. 20, no. 8, pp. 8296, 2012.

[11] C. Zuo, Q. Chen, Y. Yu, and A. Asundi, "Transport-ofintensity phase imaging using Savitzky-Golay differentiation filter-theory and applications," Optics Express, vol. 21, no. 5, pp. 5346-5362, 2013.

[12] R. W. Schafer, "What is a Savitzky-Golay filter?[lecture notes]," Signal Processing Magazine, IEEE, vol. 28, no. 4, pp. 111-117, 2011.

[13] M. Soto, E. Acosta, and S. Ríos, "Performance analysis of curvature sensors: optimum positioning of the measurement planes," Optics express, vol. 11, no. 20, pp. 2577-2588, 2003.

[14] C. E. Rasmussen, "Gaussian processes for machine learning," 2006.
[15] P. Sollich and C. K. I. Williams, "Using the equivalent kernel to understand gaussian process regression," in Advances in Neural Information Processing Systems 17. 2005, pp. 1313-1320, MIT Press.

[16] D. Paganin, A. Barty, P. J. McMahon, and K. A. Nugent, "Quantitative phase-amplitude microscopy. III. the effects of noise," J. Microsc, vol. 214, no. 1, pp. 51-61, 2004.

[17] K. A. Nugent, T. E. Gureyev, D. F. Cookson, D. Paganin, and Z. Barnea, "Quantitative phase imaging using hard X rays," Physical review letters, vol. 77, no. 14, pp. 2961-2964, 1996.

[18] J. M. Cowley, Diffraction physics, North Holland, 1995.

[19] B. Xue, S. Zheng, L. Cui, X. Bai, and F. Zhou, “Transport of intensity phase imaging from multiple intensities measured in unequally-spaced planes," Optics express, vol. 19, no. 21, pp. 20244-20250, 2011. 